\title{
MicroRaman Spectroscopy of Si Nanowires: Influence of Size
}

\author{
J. Anaya ${ }^{1}$, C. Prieto ${ }^{1}$, A. Torres ${ }^{1}$, A. Martín-Martín ${ }^{1}$, J. Souto ${ }^{1}$, \\ J. Jiménez ${ }^{19}, A$. Rodríguez ${ }^{2}$ and T.Rodríguez ${ }^{2}$ \\ ${ }^{1}$ GdS Optronlab, Dpto. Física de la Materia Condensada, Ed. I+D, Univ. de Valladolid, Paseo de \\ Belén 1, 47011 Valladolid, Spain \\ ${ }^{2}$ Tecnología Electrónica, E.T.S.I.T., Universidad Politécnica de Madrid, 28040 Madrid, Spain \\ ajimenez@fmc.uva.es
}

Keywords: Nanowires, Raman spectroscopy, Si, Temperature

\begin{abstract}
Si Nanowires (NWs) were studied by Raman microspectroscopy. The Raman spectrum of the NWs reveals important thermal effects, which broaden and shift the one phonon Raman bands. The low thermal conductivity of the NWs and the low thermal dissipation are responsible for the temperature enhancement in the NW under the excitation with the laser beam. We have modeled, using finite element methods, the interaction between the laser beam and the NWs. The Raman spectrum of Si NWs is interpreted in terms of the temperature induced by the laser beam excitation, in correlation with finite element methods (fem) for studying the interaction between the laser beam and the NWs.
\end{abstract}

\section{Introduction}

Semiconductor NWs have attracted a great deal of attention because of their potential application to new devices due to their unique optical and electrical properties [1]. The characterization of NWs must take account of their reduced size, which introduces changes in their properties with respect to their bulk counterpart. Raman spectroscopy is a powerful tool for studying the properties of structures of reduced dimension, since the shape of the Raman spectrum is sensitive to the phonon confinement associated with the reduced size [2.3]. Normally, bundles of NWs are studied, which means that a determined number of NWs can be simultaneously probed by the laser beam; therefore, the one phonon Raman band lineshape can be influenced by the dispersion of NWs dimensions. Other factors contributing to the band broadening and shift in Si NWs are Fano broadening due to photoexcited carriers [4, 5], and heating induced by the laser beam [5-7]. The Raman microprobe works with a relative high laser power density, typically up to $100 \mathrm{KWcm}^{-2}$; under this excitation the reduced thermal conductivity of the NWs does not warrant an efficient heat evacuation; therefore, the NWs can reach elevated temperatures, which broaden and downshift the one phonon Raman bands [5-7], leading to Raman band lineshapes similar to those originated from phonon confinement, which are only appreciable for NWs diamters $<20 \mathrm{~nm}$. The NWs with large diameter $(>20 \mathrm{~nm})$, should present a phonon bulk like behaviour; however, the phonon band lineshape measured does not match the one of the bare bulk material. NWs are systems with lower thermal conductivity than the bulk materials because of the surface phonon scattering [8]; furthermore, they generally stand in air, therefore, heat dissipation is very inefficient. The poor thermal transport and dissipation constitutes a serious problem for the practical use of NWs. In this context, the understanding of the interaction between the laser beam and the NWs is crucial for the interpretation of the Raman spectrum of NWs. We present herein a study of the Raman spectrum of Si NWs, revealing the influence of the laser induced heating, showing how pure thermal effects can be erroneously interpreted in terms of phonon confinement. 


\section{Experimental Set-Up}

Si NWs were grown by the vapor liquid solid (VLS) method in a low pressure chemical vapor deposition (LPCVD) reactor on (100) $\mathrm{Si}$ substrates. $\mathrm{Si}_{2} \mathrm{H}_{6}$ was the precursor gas (10 sccms). A colloidal suspension of Au particles (30 nm in diameter) was used for the catalyst particles. Typical NWs grown by this method had diameters ranging from 10 to $200 \mathrm{~nm}$ and several $\mu \mathrm{ms}$ length.

The Raman spectra were acquired with a Labram UV-HR 800 Raman spectrometer (Jobin Yvon). The excitation was done with He-Ne laser $(632.8 \mathrm{~nm})$, power in the focal of $1.8 \mathrm{~mW}$, with a Gaussian power distribution. The diameter of the laser beam spot at the focus plane is $\cong 1 \mu \mathrm{m}$, which is significantly larger than the NWs diameter, at least one order of magnitude. Both bundles of NWs anchored to the substrate, and individual NWs detached from the substrate by sonication in an ethanol suspension and dropped on an electron microscope grid holder were used for the Raman measurements.

\section{Results and Discussion}

One expects bulk like Raman spectrum for NWs with diameter larger than $20 \mathrm{~nm}$; however, the one phonon Raman band is broadened and down shifted even for NWs with diameters larger than the threshold for phonon confinement observation [3]. In this scenario, if the Raman spectra are analyzed on the basis of a phonon confinement model important errors of appreciation can be achieved. One can find in the literature complex formalisms using phonon confinement, thermal gradients, and diameter distributions for describing the Raman spectrum of NWs [9]. When NWs are excited by a laser beam, the temperature enhancement, a priori, shall depend on the NWs dimensions [7], the surrounding media [7], and the anchoring of the NW to the substrate; free standing NWs should reach higher temperatures than NWs anchored to the substrate, which acts as a heat sink.

Usually, one uses the laser beam power density as the parameter accounting for the incident energy on the sample; however, in the case of nanosized structures, e.g. NWs, their size is at least one order of magnitude smaller than the laser beam diameter at focus; therefore, the use of the laser power density as a parameter does not make sense, because the laser energy absorbed by the NW depends on its position inside the laser beam spot, which the spatial power distribution follows a Gaussian law. For example, in the case of a bundle of NWs, the laser spot focused on the sample surface is shared by several NWs, see Fig.1, with the NWs occupying different positions inside the laser beam spot, which means that all of them are not excited under the same conditions. The result of this is a Raman spectrum, which is the convolution of the spectra arising from the several NWs excited under different conditions each one.

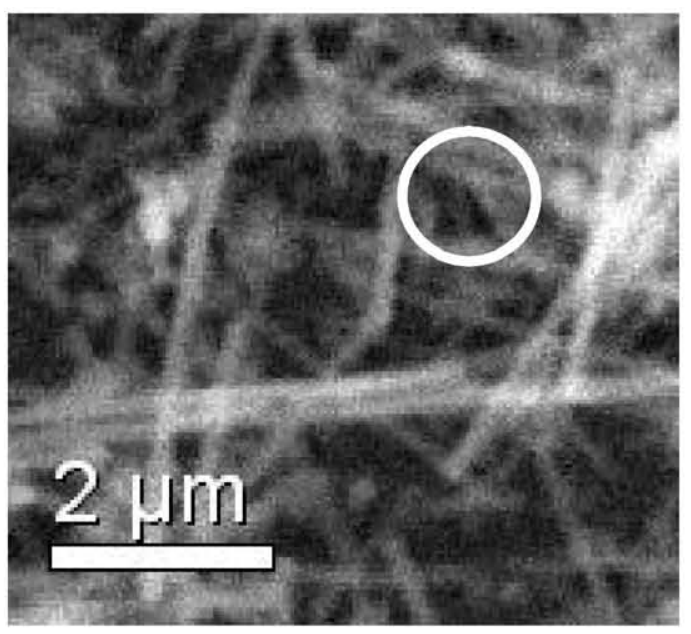

Fig.1. SEM image of NWs, the circle represents the laser beam

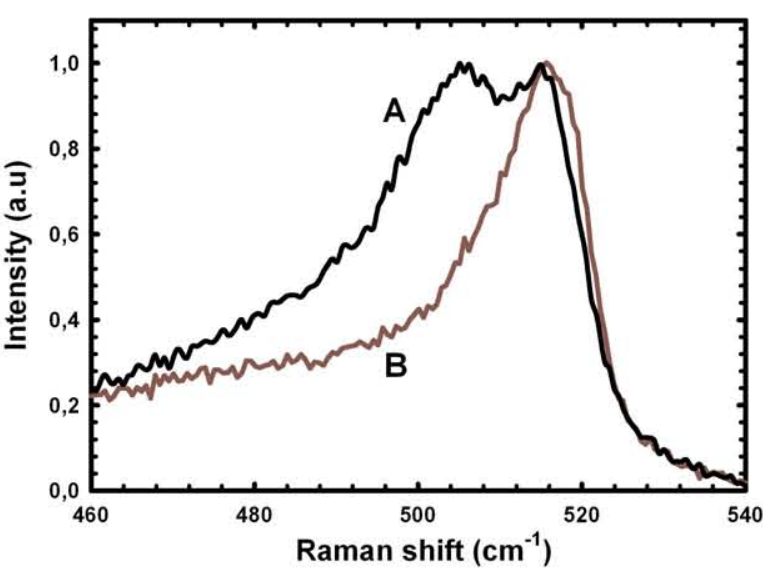

Fig.2. Typical Raman spectra measured in bundles of NWs 
Experimentally, one observes that in the absence of phonon confinement because of the large diameters the Raman bands are anomalously deformed. Two spectra measured at different positions of a bundle of NWs are shown in Fig.2. One observes clear differences between the spectra obtained for positions of the laser beam over the bundle of NWs displaced with respect to each other. The frequency shift and broadening varies depending on the different contributions of the NWs probed by the focused laser beam; note that one can even split the band. The interaction between the NWs and the laser beam can be modelled by a finite element method (fem). The main factors contributing to the NWs overheating, are the laser energy absorbed, which depends on the position of the NWs inside the laser beam spot; and the NW dimensions, both diameter and length, which determine the thermal conductivity and the heat dissipation respectively. The fem calculation permits to establish the temperature reached by a NW of a particular dimension, placed inside the laser beam spot [7]. Once the temperature is estimated one can calculate the Raman spectrum for each NW inside the laser beam spot. Fig. 3 shows the temperature reached by four NWs with the same diameter $(56 \mathrm{~nm})$ occupying different positions inside the laser beam spot, for two different positions of the laser beam. Fig. 4 shows the Raman spectra arising from each NW, and the global spectrum calculated by the addition of the four spectra weighted by their respective volume contribution.
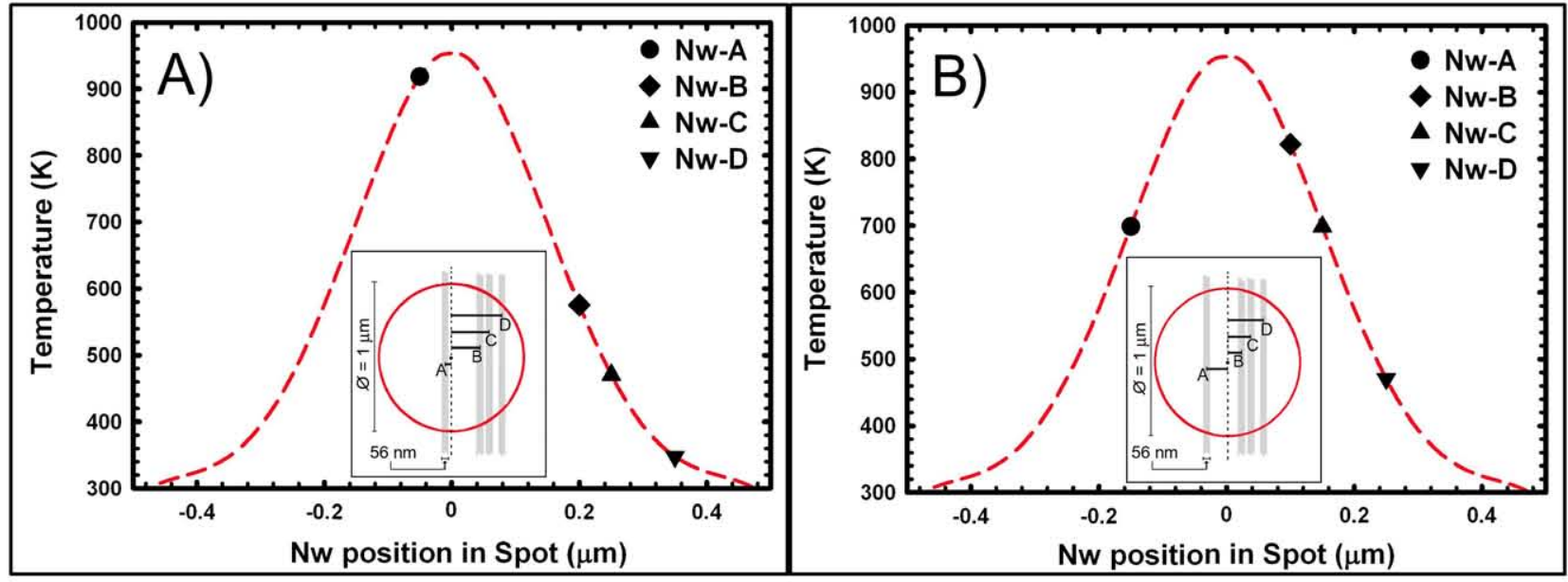

Fig.3. Temperature reached by the four NWs disposed as shown in the insets, for two different positions, $\mathrm{A}$ and $\mathrm{B}$, of the laser beam.

In a pure phonon confinement model, the global spectra can be fitted with a reduced diameter size, $<10 \mathrm{~nm}$, which would give an erroneous interpretation of the Raman spectrum. Note that the results obtained should depend on the relative position of the four NWs, which cannot be determined in the optical microscope, because of the small dimension, below the optical resolution, of the NWs, which do not permit the observation of their exact position. See that for certain poitions of the NWs one reproduces spectra similar to the experimental ones represented in Fig.2; this is only an approximate similarity, since the distribution of the NWs inside the laser beam in the experimental case is not known, therefore, it cannot be exactly correlated to the simulated configuration, Fig.3. Anyway, the information that one can extract from the Raman spectrum of a bundle of NWs is very ambiguous. To relieve this problem one needs to work with individual NWs. 

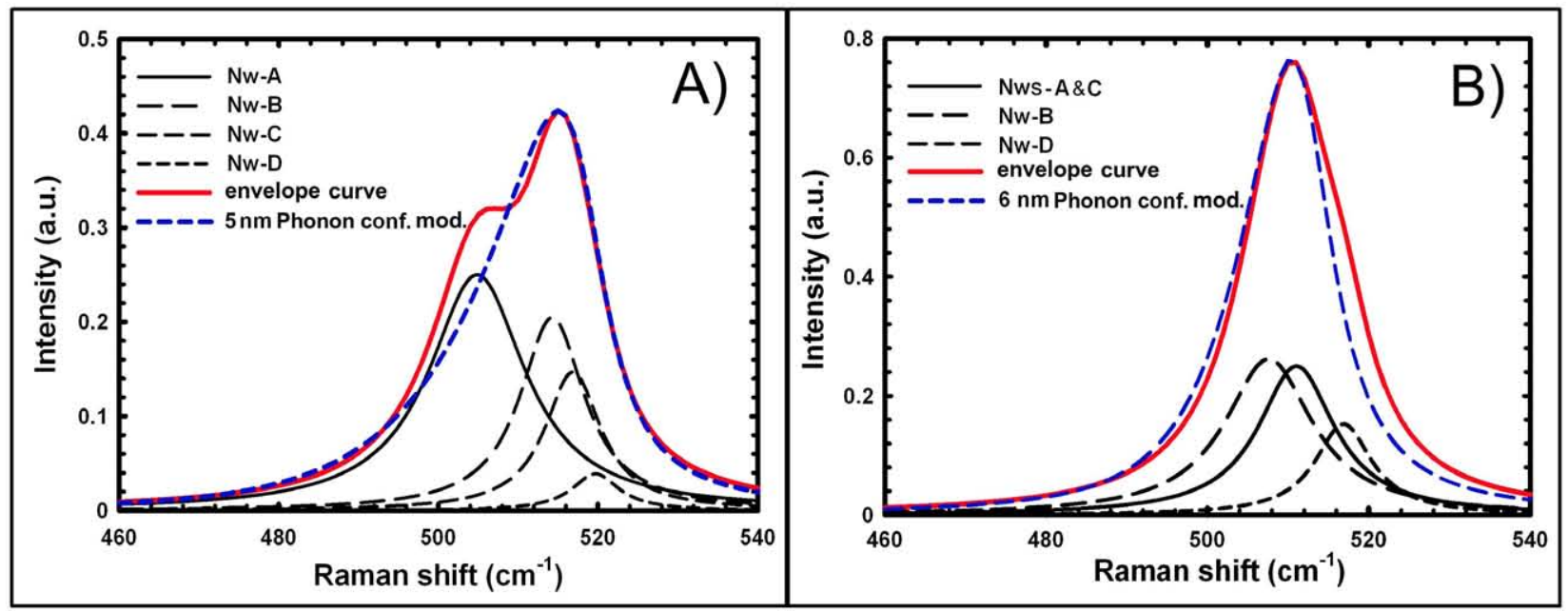

Fig. 4. Calculated Raman spectrum for each NW, and global spectra of the four NWs, in the two positions of the NWs, A and B respectively.

\section{Conclusion}

The Raman spectrum of NWs is very difficult to interpret when several NWs are simultaneously measured by the laser beam; however, the measurement of the spectrum of individual NWs relieves the ambiguity of the Raman spectrum interpretation. According to our discussion, one could use Raman micro-spectroscopy combined with fem analysis as a non destructive tool for studying thermal transport in NWs.

\section{Acknowledgments}

This work was funded by the Spanish Government (MAT-2007-66181 and MAT-2010-20441) and by Junta de Castilla y León (VA051A06 -GR202).

\section{References}

[1]. Y.Cui and C.M.Lieber, Science, 291, 851 (2001).

[2]. H. Richter, Z.P. Wang and L. Ley, Solid St. Commun. 39, 625 (1981).

[3]. I. H. Campbell and P.M.Fauchet, Solid St. Commun. 58, 739 (1986).

[4]. V.Magidson, R.Beserman; Phys.Rev.B 66, 195206 (2002)

[5]. S. R. Gupta, Q. Xiong, C. K. Adu, U. J. Kim, and P. C. Eklund, Nano Lett. 3, 627 (2003).

[6]. H. Scheel, S. Reich, A.C. Ferrari, M. Cantoro, A. Colli, and C. Thomsen. Appl. Phys. Lett. 88, 233114, (2006)

[7]. A. Torres, A. Martín-Martín, O. Martínez, A.C. Prieto, V. Hortelano, J. Jiménez, A. Rodríguez, J. Sangrador and T. Rodríguez, Appl. Phys. Lett 96, 011904 (2010).

[8]. D.Li, Y.Wu, P.Kim, L.Shi, P.Yang and A.Majumdar, Appl.Phys.Lett., 83, 2934 , (2003).

[9]. K. W. Adu, H. R. Gutiérrez, U. J. Kim, and P. C. Eklund, Phys.Rev. B 73, 155333 (2006). 